\title{
Rôle physiologique de la $\gamma$-glutamyl transpeptidase
}

La $\gamma$-glutamyl transpeptidase ( $\gamma \mathbf{G T})$ n'est souvent connue que comme un marqueur de diverses maladies hépatiques. Elle est aussi une enzyme essentielle du cycle du glutathion entre différents organes, principalement le foie, le rein et l'intestin. Elle intervient dans la détoxification de nombreux xénobiotiques et dans le métabolisme des leucotriènes. Les modifications pathologiques de l'activité de cette enzyme pourraient donc moduler le métabolisme du glutathion et de ses dérivés.

\section{Georges Guellaën}

\section{ADRESSE}

G. Guellaën : directeur de recherche à l'Inserm. Inserm U. 99, hôpital Henri-Mondor. 94010 Créteil, France.

$\mathrm{m} / \mathrm{s} n^{\circ} 9$ vol. 5, novembre 89

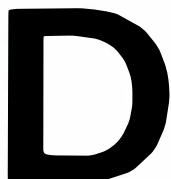

ans la plupart des esprits., "avoir les $\gamma \mathrm{GT}$ élevées » est associé avec «maladie du foie », ce qui se traduit rapidement pour certains en « penchant marqué pour l'alcool ». Il est vrai qu'une élévation de gamma-glutamyl transpeptidase $(\gamma \mathrm{GT})$ sérique est le plus souvent associée avec un trouble hépatique, mais la nature peut en être très variée. Quelques maladies graves et peu fréquentes, comme les cancers du foie, sont parfois à l'origine d'une augmentation de $\gamma \mathrm{GT}$ sérique. Dans la majorité des cas, les atteintes sont bénignes. De nombreux composés, absorbés ou inhalés (alcool, médicaments, solvants) peuvent provoquer une telle élévation et, à ce titre, la $\gamma \mathrm{GT}$ représente un des meilleurs marqueurs de l'alcoolisme chronique. Que le lecteur ayant un taux de $\gamma \mathrm{GT}$ « au plafond» se rassure, il a encore toutes ses chances; $5 \%$ de la population a une $\gamma \mathrm{GT}$ élevée asymptomatique.

Bien évidemment, la Nature ne nous a pas dotés d'une panoplie de marqueurs « aide au diagnostic » afin de dépister nos maladies ou... nos faiblesses. Dans l'organisme, ces marqueurs jouent un rôle et celui-ci est le plus souvent méconnu. C'est le cas de la $\gamma$ GT. Si d'aventure le lecteur veut s'informer sur le rôle de cette enzyme, sa quête sera difficile. Il restera perplexe en consultant les différents manuels de physiologie humaine ou de biochimie. Dans ces livres, lorsqu'elle est citée, la $\gamma \mathrm{GT}$ a un «rôle physiologique inconnu », et dans le meilleur des cas elle « catalyse le transfert du radical glutamyl du glutathion sur un acide aminé ». Dans cette revue, nous allons tenter de préciser ce domaine. Après avoir rappelé quelques données essentielles sur la $\gamma \mathrm{GT}$ et ses régulations, nous ferons le point sur l'importance des différentes voies métaboliques dans lesquelles est engagée cette enzyme chez les mammifères.

Réactions catalysées par la $\gamma \mathbf{G T}$

La $\gamma \mathrm{GT}$ catalyse plusieurs réactions du métabolisme du glutathion (GSH) et de ses dérivés [1, 2]. Le GSH 


\section{RÉFÉRENCES}

l. Curthoys NP, Hughey R. Characterization and physiological function of rat renal $\gamma$ glutamyl transpeptidase. Enzyme 1979; 24 383-403.

2. Goldberg DM. Structural, functionnal and clinical aspects of $\gamma$-glut amyltranferase. CRC Crit Rev Clin Lab Sci 1980 ; 12 : 1-58.

3. Meister A, Anderson ME. Glutathione. Ann Rev Biochem 1983 ; 52: 11-60.

4. Pfaller W, Gstraunthaler G, Kotanko P Wolf $\mathrm{H}$, Curthoys NP. Immunocytochemical localization of $\gamma$-glutamyl transferase on isolated renal cortical tubular fragments. Histochemistry $1984 ; 80$ : 289-93.

5. Braun JP, Rico AG, Benard P, BurgatSacaze Y. La $\gamma$-glutamyltransférase. Ann Biol Clin $1977 ; 35: 433-57$.

6. Finidori J, Laperche $\mathrm{Y}$, Haguenauer-Tsapis R, Barouki R, Guellaen G, Hanoune J. In vitro biosynthesis and membrane insertion of $\gamma$-glutamyltranspeptidase. J Biol Chem $1984 ; 259$ : 4687-90.

7. Barouki R, Finidori J, Chobert MN, Aggerbeck M, Laperche Y, Hanoune J. Biosynthesis and processing of $\gamma$-glutamyl transpeptidase in hepatoma tissu culture cells. Biol Chem 1984; 259 : 7970-4.

8. Laperche $\mathrm{Y}$, Bulle $\mathrm{F}$, Aissani $\mathrm{T}$, et al. Molecular cloning and nucleotide sequence of rat kidney $\gamma$-glutamyl transpeptidase cDNA. Proc Natl Acad Sci USA 1986; 83 $937-41$

9. De Meyts E, Heisterkamp N, Groffen J. Cloning and nucleotide sequence of human $\gamma$-glutamyl transpeptidase. Proc. Natl Acad Sci USA $1988 ; 85: 8840-4$

10. Sakamuro D, Yamazoe M, Matsuda Y, et al. The primary structure of human $\gamma$-glutamyl transpeptidase. Gene $1988 ; 73$ : 1-9.

11. Goodspeed DC, Dunn DJ, Miller CD, Pitot H. Human $\gamma$-glutamyl transpeptidase cDNA : comparison of hepatoma and kidney mRNA in the human and rat. Gene 1989 ; 76 : $1-9$.

12. Pawlak A, Lahuna O, Bulle F, et al. $\gamma$ glutamyl transpeptidase : a single copy gene in the rat and a multigene family in the human genome. J Biol Chem ; 263 : 9913-9.

13. Bulle F, Mattei MG, Siegrist S, et al. Assignement of the human $\gamma$-glutamyl transferase gene to the long arm of chromosome 22. Hum Genet 1987; 76 : 283-6.

14. Laperche Y, Guellaen G, Barouki R, Hanoune J. Biosynthesis and regulation of $\gamma$ glutamyl transpeptidase. In: Visa J, ed. Handbook of glutathion: metabolism and physiological functions. Boca Raton: CRC

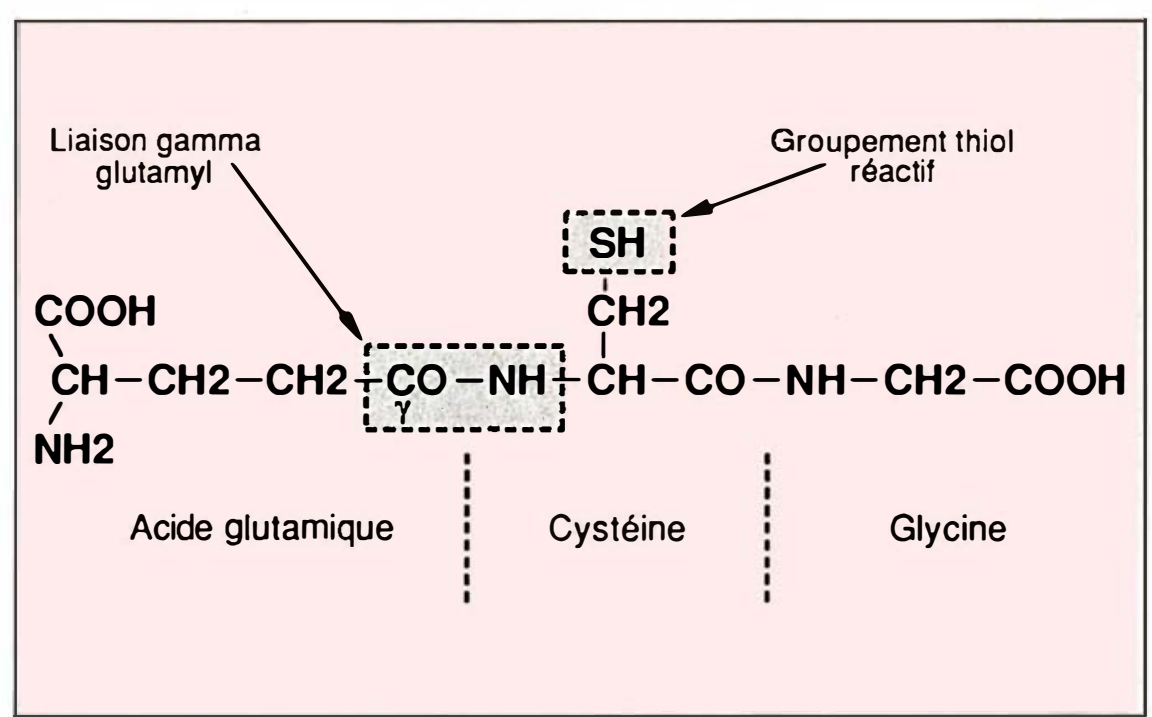

Figure 1. Structure du glutathion.

est une petite molécule ( $\gamma$-Glu-CysGly) (figure 1) représentant le composé thiol $(\mathrm{SH})$ le plus abondant de l'organisme. Il est caractérisé par une liaison peptidique particulière entre le $\mathrm{COOH}$ en position $\gamma$ de l'acide glutamique et la cystéine, ce qui le rend résistant à certaines protéases. Le GSH est essentiel pour la détoxification cellulaire, dans l'équilibre de certaines réactions d'oxydoréduction, et il représente le réservoir de cystéine le plus important pour les tissus [3].

$\mathrm{Au}$ cours de ces réactions, le GSH subit différentes transformations et la $\gamma \mathrm{GT}$ est souvent le premier élément à intervenir dans le cours de ces modifications. Elle seule est capable de couper cette liaison peptidique spécifique du GSH et de ses dérivés. Cette coupure peut se faire suivant trois types de réactions (figure 2). Les différentes voies métaboliques où surviennent ces réactions seront détaillées dans le chapitre consacré au rôle de cette enzyme.

\section{Localisation et structure de la $\gamma G T$}

La $\gamma \mathrm{GT}$ est présente à la face externe des cellules d'organes très actifs dans le transport ou la sécrétion de métabolites. Cette enzyme, particulièrement abondante dans le rein $(2 \mathrm{U} / \mathrm{mg}$ protéine), est localisée sur la face tubulaire, et dans une moindre mesure au pôle basal, des cellules du tube contourné proximal [4]. Les autres tissus ont des activités plus faibles, c'est-à-dire en pourcentage de l'activité rénale: pancréas 20 , vésicules séminales 4 , intestin 3,3 , foie 0,2 , rate 0,2 [5]. Ces valeurs représentent l'activité moyenne par organe mais, dans certains tissus, la $\gamma \mathrm{GT}$ est concentrée dans un nombre limité de cellules. C'est le cas du foie adulte, où la $\gamma \mathrm{GT}$ est uniquement présente dans l'épithélium biliaire, et du cerveau, où l'endothélium capillaire et les plexus choroïdes sont riches en $\gamma \mathrm{GT}$.

La solubilisation, la purification et l'obtention d'anticorps contre la $\gamma \mathrm{GT}$ ont permis de déterminer la structure et les différentes étapes de la synthèse cellulaire de cette enzyme. La protéine rénale, solubilisée par un détergent non ionique (Triton X100) est composée de deux sousunités glycosylées de poids moléculaires moyens 27000 et 51000 . La $\gamma \mathrm{GT}$ rénale, détachée de la membrane par protéolyse ménagée à la papaïne, a une petite sous-unité identique (27000 Da) mais une grosse sous-unité de poids moléculaire plus faible (48 000). Cette observation a suggéré que l'ancrage de la $\gamma \mathrm{GT}$ à la membrane plasmique se faisait par l'intermédiaire de la grosse sous-unité. En outre, des expériences de marquage d'affinité ont permis de localiser le site cataly- 
tique de l'enzyme sur la petite sousunité.

Les deux sous-unités de la $\gamma \mathrm{GT}$ proviennent d'un précurseur commun de 63000 Da [6]. Par des expériences de synthèse in vitro, nous avons montré que la glycosylation de ce précurseur, et des deux sous-unités, débute dans le réticulum endoplasmique et se poursuit dans le corps du Golgi [7]. Cette glycosylation est plus riche en acide sialique dans les tissus fotaux et tumoraux que dans les tissus adultes normaux. Cette observation est à la base de l'existence de formes modifiées (isoformes) de la $\gamma \mathrm{GT}$, spécifiques de certaines affections.

\section{Clonage du gêne de la $\gamma \mathbf{G T}$}

Au laboratoire, nous avons réalisé le clonage de l'ADN complémentaire (ADNc) de cette protéine par criblage d'une banque ADNc de rein de rat avec des oligonucléotides marqués et spécifiques de la séquence protéique $\mathrm{N}$-terminale de la grosse sous-unité de la $\gamma \mathrm{GT}$.

La séquence nucléotidique des clones sélectionnés nous a permis de déterminer la séquence du précurseur de la $\gamma$ GT (568 acides aminés). Celui-ci est clivé en deux sous-unités de 379 acides aminés (4l $766 \mathrm{Da}$ ) et 189 acides aminés (20 $052 \mathrm{Da})$. L'analyse des séquences obtenues a confirmé l'ancrage de la $\gamma \mathrm{GT}$ à la membrane plasmique par la grosse sous-unité et démontré que le segment peptidique hydrophobe d'ancrage est constitué d'une séquence tout à fait particulière, le peptide signal $\mathrm{NH}_{2}$-terminal non coupé [8]. Cette séquence sert à la cellule pour identifier les protéines destinées à être membranaires ou exportées. Dans la majorité des cas, cette séquence est éliminée, les protéines membranaires restent ancrées à la membrane par l'intermédiaire d'une autre portion hydrophobe de la protéine. Dans le cas de la $\gamma \mathrm{GT}$, il n'y a pas d'autre séquence d'ancrage possible. La coupure du peptide signal désolidariserait la $\gamma \mathrm{GT}$ de la membrane, qui deviendrait ainsi une protéine sécrétée. Il y a là une voie d'investigation intéressante pour l'analyse des mécanismes potentiels de passage de la $\gamma \mathrm{GT}$ dans le sérum $\mathrm{m} / \mathrm{s} n^{\circ} 9$ vol. 5 , novembre 89 au cours des maladies hépatiques. Grâce aux sondes ADNc de $\gamma \mathrm{GT}$ de rein de rat, la $\gamma \mathrm{GT}$ a été clonée dans différents tissus humains comme le placenta, le foie foetal ou les cellules HepG2 [9-11]. Dans tous les cas, la séquence du précurseur comporte 569 acides aminés et une forte homologie $(79 \%$ ) avec la séquence $\gamma \mathrm{GT}$ de rein de rat. Les gènes humains ont été isolés au laboratoire avec ces mêmes sondes. Nous avons ainsi montré que, chez l'homme, la $\gamma \mathrm{GT}$ est codée par une famille multigénique d'au moins quatre gènes, ou pseudogènes, ce qui contraste avec le rat où un seul gène est présent [12]. Il sera intéressant de déterminer si l'expression de ces gènes peut être spécifiquement modifiée au cours de certaines maladies. En collaboration avec M.G. Mattei (Inserm U. 242),

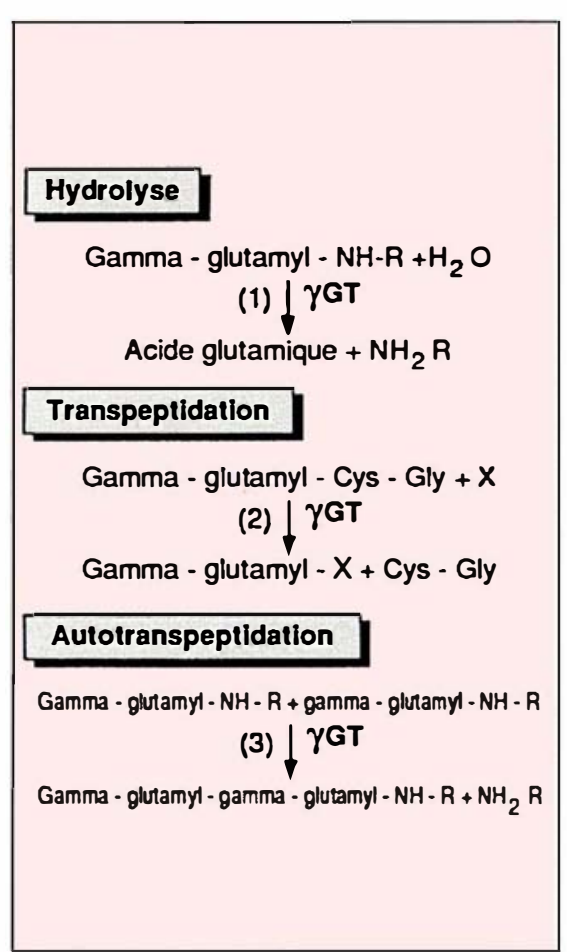

Figure 2. Réactions catalysées par la $\gamma$ GT. La réation d'hydrolyse (1) intervient dans la dégradation du GSH et de la glutamine. La transpeptidation (2) transfère le radical $\gamma$-glutamyl sur un accepteur $X$ qui peut être un acide aminé, un peptide ou un autre composé comme les leucotriènes. Cette réaction est fréquente. L'autotranspeptidation (3) fait interagir deux composés $\gamma$ glutamyl, cette réaction est plus rare et ne sera. pas développée dans cet article. nous avons localisé, par hybridation in situ, l'ensemble des gènes humains de la $\gamma \mathrm{GT}$ sur le chromosome 22 en ql1.1-q11.2[13]. Cette localisation donne à ces gènes un intérêt particulier : en effet ils représentent de nouveaux marqueurs d'une région impliquée dans de nombreux réarrangements chromosomiques associés à des maladies comme les leucémies chronique ou aiguë, le lymphome de Burkitt ou le sarcome d'Ewing (figure 3, p. 640).

\section{Régulations de la $\gamma$ GT}

L'activité de la $\gamma \mathrm{GT}$ est contrôlée dans de nombreuses situations physiologiques ou pathologiques. Nous rappellerons quelques données sur les modifications d'activité de la $\gamma \mathrm{GT}$ hépatique, l'ensemble des régulations de cette enzyme ayant fait l'objet d'une autre revue générale [14].

Dans le foie, les modifications d'activité de la $\gamma \mathrm{GT}$ peuvent survenir dans les hépatocytes ou dans l'épithélium biliaire. Dans les hépatocytes, l'activité de la $\gamma \mathrm{GT}$ varie au cours du développement. Elle chute de dix fois à la naissance pour devenir difficilement détectable chez l'adulte. A ce stade, la $\gamma \mathrm{GT}$ peut être stimulée par différents facteurs endogènes ou exogènes comme les hormones (glucocorticoïdes, hormones thyroïdiennes) ainsi que certains facteurs de croissance ou de différenciation (EGF, epidermal growth factor; acide rétinoïque). Des cellules d'origine hépatique transfectées avec l'oncogène ras expriment fortement la $\gamma \mathrm{GT}$ [15]. De nombreux agents pharmacologiques (barbituriques) provoquent également une augmentation de l'activité de cette enzyme. Les effets de l'alcool ont été largement étudiées[16] en relation avec les augmentations de $\gamma \mathrm{GT}$ sérique observées chez les alcooliques chroniques. En général tous les effets observés sont dépendants de l'état de différenciation cellulaire.

La régulation de l'activité de la $\gamma \mathrm{GT}$ hépatique au cours de la carcinogenèse est la mieux documentée[17]. La $\gamma \mathrm{GT}$ hépatique peut augmenter de 100 fois dans les cellules tumorales et dans les foyers prénéoplasiques. Nous avons déjà mentionné que ces 


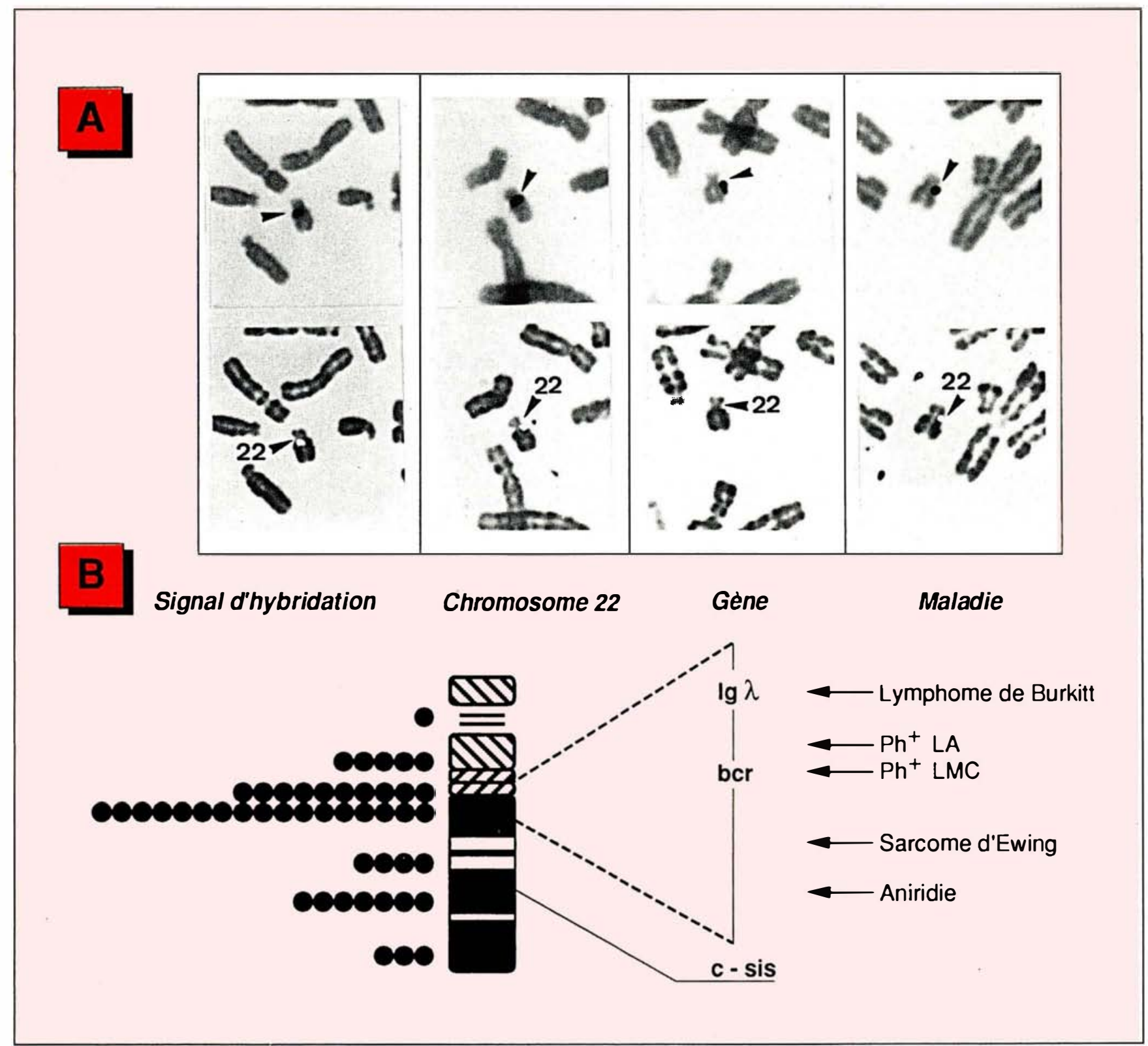

Figure 3. Localisation des séquences spécifiques de la yGT sur le chromosome 22. La sonde spécifique de la

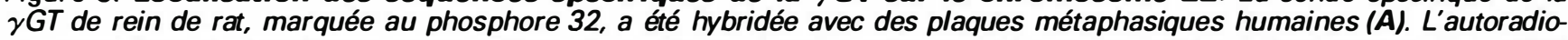
gramme d'une de ces plaques, après coloration au Giemsa (haut) et mise en évidence des bandes $R$ (bas), permet de localiser les grains d'argent sur le chromosome 22. (B) La distribution des grains d'argent sur l'ensemble des chromosomes 22 étudiés permet de positionner les gènes de la $\gamma$ GT en 22 q11.1-q11.2. D'autres gènes ont été localisés dans cette région, comme Ig $\lambda$ (partie constante des chaînes légères des immunoglobulines) et bcr (breakpoint cluster region) ainsi que les points de

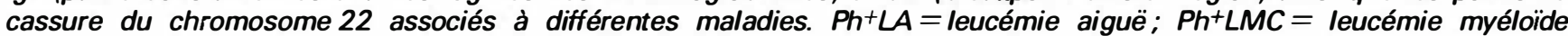
chronique $; \mathrm{Ph}^{+}=$chromosome philadelphie.

variations d'activité peuvent être associées à une modification de la glycosylation et à un passage de la $\gamma \mathrm{GT}$ dans le sang. Les mécanismes responsables de ces augmentations sont inconnus, mais ils ne sont pas liés à la prolifération cellulaire. En effet, certains composés, comme les agents dits « proliférateurs de peroxi- somes ", peuvent induire des tumeurs hépatiques dépourvues d'activité $\gamma \mathrm{GT}$. Par ailleurs, l'augmentation d'activité de la $\gamma \mathrm{GT}$, décrite par certains auteurs au cours de la régénération hépatique, n'est observable que sur certaines souches d'animaux.

D'autres maladies modifient l'activité de la $\gamma \mathrm{GT}$ des cellules biliaires.
C'est le cas de la plupart des cholestases intra- ou extrahépatiques. A partir de différents modèles expérimentaux de ces affections, nous avons observé une prolifération cholangiolaire riche en $\gamma \mathrm{GT}$ à l'origine d'une augmentation d'activité sérique. Seules certaines cholestases, comme la maladie de Byler [18] ou 
les cholestases récurrentes bénignes, gardent un taux normal de $\gamma \mathrm{GT}$ sérique.

Dans le rein, l'activité de la $\gamma \mathrm{GT}$ est 1000 fois plus élevée que dans le foie, mais, en général, les lésions rénales sont caractérisées par une augmentation de la $\gamma \mathrm{GT}$ urinaire. Jusqu'à présent aucune corrélation n'a été établie entre des maladies rénales et une augmentation de la $\gamma \mathrm{GT}$ sérique, si l'on exepte les rejets de greffe.

\section{Rôle métabolique de la $\gamma$ GT}

La $\gamma \mathrm{GT}$ participe à plusieurs voies métaboliques. Nous examinerons successivement le rôle de la $\gamma \mathrm{GT}$ dans le cycle du GSH et le métabolisme interorgane de ce composé, l'implication éventuelle de la $\gamma \mathrm{GT}$ dans le transport des acides aminés, l'hydrolyse de la glutamine, la détoxification cellulaire et enfin la place de la $\gamma \mathrm{GT}$ dans le métabolisme des leucotriènes.

La $\gamma \mathrm{GT}$ joue un rôle majeur dans le cycle du GSH. Ce cycle caractérisé par Meister [3] est représenté sur la figure 4. La cystéine est couplée à l'acide glutamique, par la $\gamma$-glutamyl cystéine synthétase, le dipeptide $\gamma$-Glu-Cys formé est transformé en GSH par la glutathion synthétase en y ajoutant la glycine. Le GSH synthétisé sort de la cellule par l'intermédiaire d'un transporteur. Il est hydrolysé par la $\gamma \mathrm{GT}$ située à la face externe de la membrane plasmique de la même cellule ou d'un autre organe. Les produits de la réaction, l'acide glutamique et le dipeptide Cys-Gly, hydrolysé par une dipeptidase, sont recaptés par la cellule et participent à nouveau à la synthèse de GSH. Dans le cas d'une réaction de transpeptidation, que nous analyserons ultérieurement, le glutamate est régénéré à partir du dérivé $\gamma$ glutamyl acide aminé (figure 4).

Quelle est l'importance de ce cycle? Le point majeur réside dans l'incapacité de toutes les cellules, sauf les cellules hépatiques, à synthétiser la cystéine. En effet les cellules du foie ont la propriété de synthétiser la cystéine à partir de la méthionine. Elles ont ainsi un avantage remarquable permettant au foie d'être le principal producteur de GSH [19]. Les autres cellules, pour subvenir à $\mathrm{m} / \mathrm{s} n^{\circ} 9$ vol. 5 , novembre 89

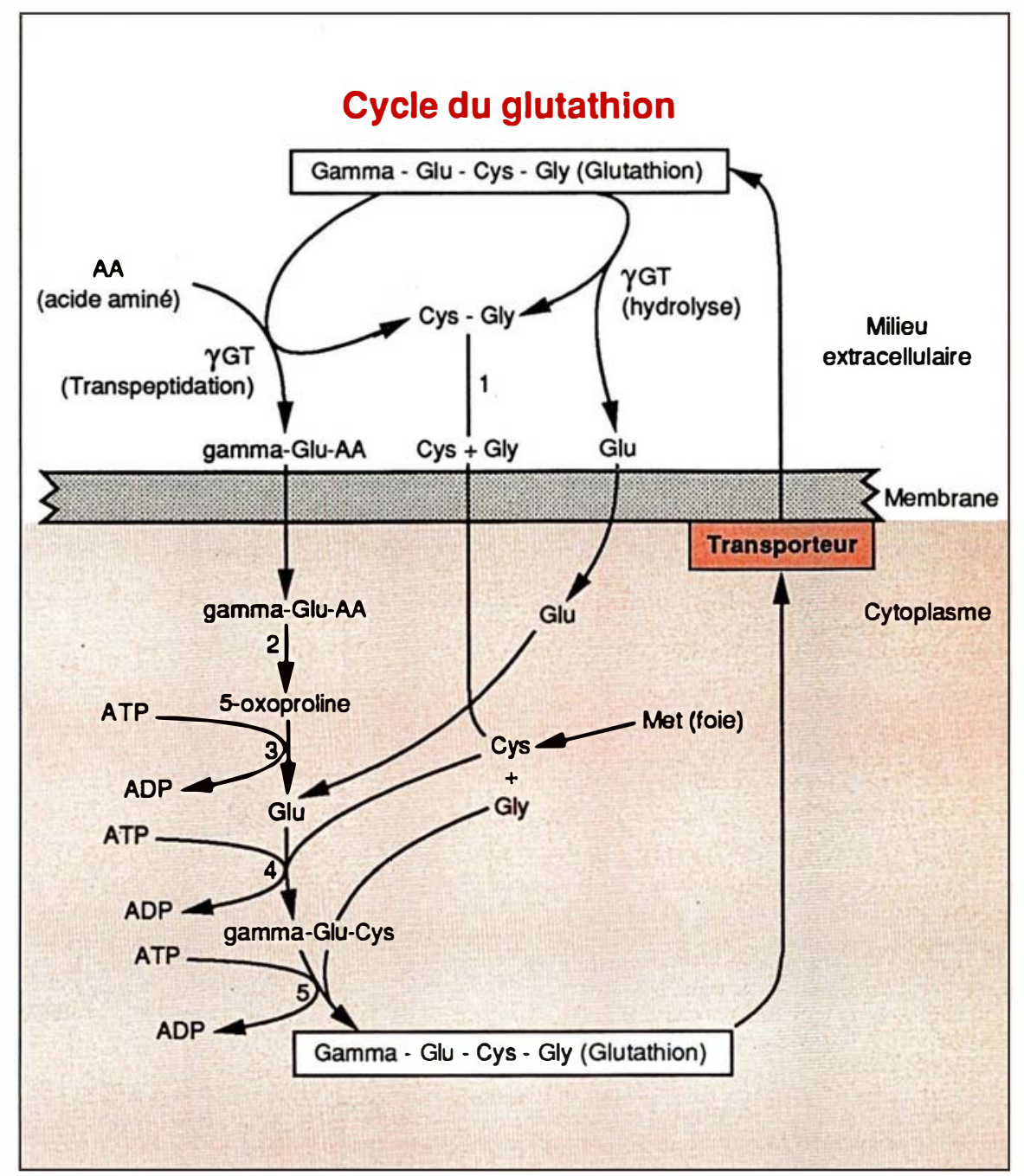

Figure 4. Cycle du glutathion. La voie impliquant une transpeptidation est catalysée par une dipeptidase (1), une $\gamma$-glutamyl cyclotransférase (2), une 5oxoprolinase (3) une $\gamma$-glutamyl cystéine synthétase (4) et une glutathion synthétase (5). Cette voie consomme trois molécules d'ATP. La réaction d'hydrolyse met en jeu les enzymes (1), (4) et (5) et n'utilise que deux molécules d'ATP.

leurs besoins, sont obligées de récupérer la cystéine présente dans le sang. Or celle-ci est principalement sous forme de GSH, synthétisé par le foie et libéré dans la circulation générale. Pour récupérer cette cystéine présente dans le GSH, il y a deux possibilités. Le GSH peut entrer directement dans les cellules par l'intermédiaire d'un transporteur, mais cette voie, peu efficace, ne contribue qu'à $15 \%$ de la captation de GSH. En revanche, le GSH est rapidement hydrolysé par les tissus contenant de la $\gamma \mathrm{GT}$, comme le rein, où $80 \%$ du GSH est dégradé en un seul passage, et l'intestin qui récu- père le GSH libéré dans la bile. Les acides aminés libérés sont captés par la cellule. En conclusion, le GSH sert de transporteur de cystéine entre le foie et les autres organes et la $\gamma \mathrm{GT}$ permet l'entrée de cette cystéine dans la cellule [20].

Ce transport de cystéine sous forme de GSH présente un avantage par rapport à la cystéine libre. En effet dans le sang, la cystéine s'oxyde rapidement en cystine. Or, la cystine entre dans les cellules par l'intermédiaire d'un transporteur indépendant du cation $\mathrm{Na}^{+}$, peu efficace, qui n'a été mis en évidence jusqu'à présent que sur les fibroblastes et les 


\section{RÉFÉRENCES}

15. Sinha S, Marshall CJ, Neal GE. $\gamma$-glutamyl transpeptidase and the ras- induced transformation of a rat liver cell line. Cancer Res 1986 ; 46 : 1440-5.

16. Barouki R, Chobert MN, Finidori J, Aggerbeck M, Nalpas B, Hanoune J. Ethanol effects in a rat hepatoma cell line: induction of $\gamma$-glutamyl transpeptidase. Hepatology $1983 ; 3$ : 323-9.

17. Hanigan $\mathrm{MH}$, Pitot $\mathrm{H}$. $\gamma$-glutamyl transpeptidase, its role in hepatocarcinogenesis. Carcinogenesis 1985 ; 6 : 165-172.

18. Chobert MN, Bernard O, Bulle F. et al High hepatic $\gamma$-glutamyl transpeptidase $(\gamma \mathrm{GT})$ activity with normal serum $\gamma \mathrm{GT}$ in children with progressive idiopathic cholestasis. J Hepatol $1989 ; 8$ : 22-5.

19. Kaplowitz N, Aw TY, Ookhtens M. The regulation of hepatic glutathione. Ann Rev Pharmacol Toxicol 1985; 25 : 715-44.

20. Inoue $M$. Interorgan metabolism and membrane transport of glutathione and related compound. In : Kuine RKH, ed. Renal Biochemistry Amsterdam: Elsevier Sciences Publ, 1985 : 225-69.

21. Bannai S, Tateishi N. Role of membrane transport in metabolism and function of glutathione in mammals. J Membrane Biol $1986 ; 89: 1-8$.

22. Ahmad S, Okine L, Wood R, Aljian J, Vistica DT. $\gamma$-glutamyl transpeptidase and maintenance of thiol pools in tumor cells resistant to alkylating agents. J Cell Physiol $1987 ; 131: 240-6$.

23. Cornell J, Meister A. Glutathione and $\boldsymbol{\gamma}$ glutamyl cycle enzymes in crypt and villus tip cells of rat jejunal mucosa. Proc Natl Acad Sci USA 1976 ; 73 : 420-22.

24. Ishii T, Sugita Y, Bannai S. Regulation of glutathione levels in mouse spleen lymphocytes by transport of cysteine. J Cell Physiol 1987 ; $133: 330-36$.

25. Meister A. On the enzymology of aminoacid transport. Science 1973 ; 180 : 33-39.

26. Griffith OW, Bridges RJ, Meister A Transport of $\boldsymbol{\gamma}$-glutamyl amino-acids : role of glutathione and $\gamma$-glutamyl transpeptidase. Proc Natl Acad Sci USA 1979 ; 12 : 6913-22.

27. Hsu BYL, Foreman JW, Corcoran SM, Ginkinger K, Segal S. Absence of a role of $\boldsymbol{\gamma}$ glutamyl transpeptidase in the transport of amino acids by rat renal brushborder membrane vesicles. J Membrane Biol 1984; 80 : hépatocytes. La situation est plus favorable pour le GSH. Ce composé s'oxyde moins rapidement que la cystéine et la cystéine, libérée à proximité de la membrane plasmique par l'hydrolyse du GSH, entre dans la cellule avant toute oxydation, grâce à un transporteur ubiquitaire d'acide aminé neutre (ASC) dépendant du $\mathrm{Na}^{+}[21]$.

L'importance de la $\gamma \mathrm{GT}$ est donc évidente dans l'approvisionnement des cellules en cystéine. Doit-on en conclure que toute cellule ayant une forte activité $\gamma \mathrm{GT}$ possède un taux élevé de GSH ?
C'est le cas de différentes cellules tumorales qui ont un taux de GSH élevé, en corrélation avec l'activité de la $\gamma \mathrm{GT}$ membranaire. Ces taux élevés de GSH rendraient ces cellules résistantes à certains agents destinés à les détruire [22]. Néanmoins cette observation n'est pas systématique. Les taux de GSH et de $\gamma \mathrm{GT}$ dans les villosités des cellules intestinales ont une corrélation inverse [23]. Par ailleurs, certaines cellules ayant des activités $\gamma \mathrm{GT}$ différentes peuvent avoir un taux de GSH identique. En fait, plusieurs composants participent à cette régulation. La $\gamma \mathrm{GT}$

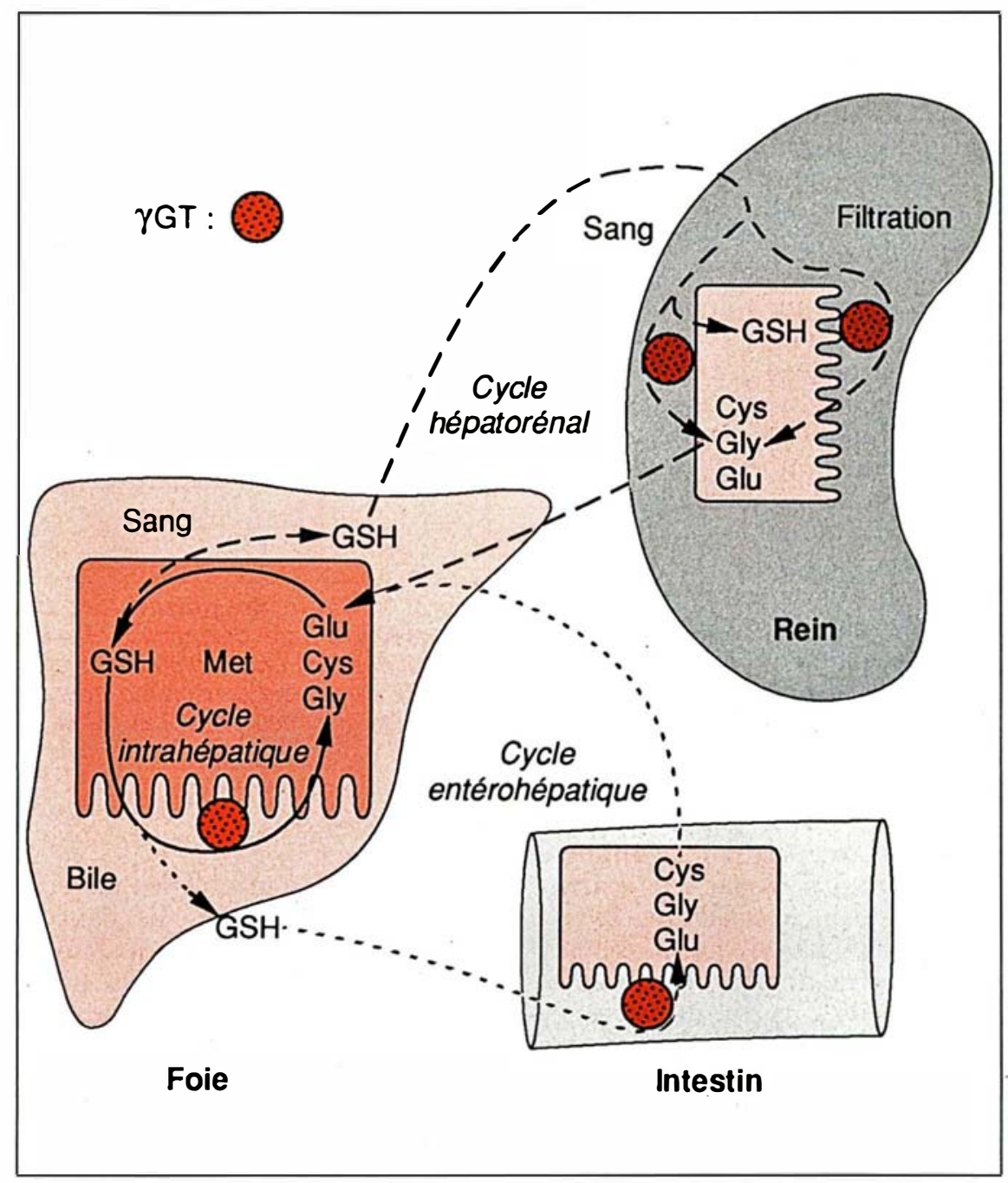

Figure 5. Métabolisme inter-organe du glutathion. Le GSH est principalement synthétisé dans le foie suivant trois cycles. Un cycle intrahépatique (-), où le GSH est hydrolysé par la yGT au pôle biliaire des hépatocytes ou de l'épithélium biliaire. Un cycle entérohépatique (......) qui récupère le GSH arrivé dans l'intestin. Un cycle hépatorénal (------) qui récupère la majorité (80\%) du GSH libéré à la face sinusoidale des hépatocytes. Dans le rein, le GSH est hydrolysé à la face sinusoïale (30\%) ou luminale (70\%) des cellules du tube contourné proximal. L'existence d'un transport direct du GSH dans la cellule rénale est encore discutée. 
favorise l'apport des précurseurs du GSH, mais la présence du transporteur de la cystéine [24], et des autres enzymes du métabolisme du GSH, est indispensable. En outre, la synthèse de GSH nécessite deux ATP (trois pour la transpeptidation), et certaines cellules peuvent ne pas disposer d'une telle énergie. En conclusion, la $\gamma \mathrm{GT}$ seule ne suffit pas à maintenir un taux intracellulaire élevé de GSH, mais elle participe de manière active à l'approvisionnement et au renouvellement de ce composé.

Le transport d'acide aminés a été longtemps considéré comme un des rôles majeurs de la $\gamma \mathrm{GT}[25,26]$. Ce transport intervient dans le cycle du GSH lors de la transpeptidation (figure 5). Le complexe cystéineacide aminé entre dans la cellule, est hydrolysé par la $\gamma$-glutamyl cyclotransférase libérant ainsi un acide aminé. Certaines expériences d'introduction de $\gamma \mathrm{GT}$ purifiée dans des érythrocytes dépourvus de cette enzyme, ou dans des vésicules de lécithines, ont montré que la $\gamma \mathrm{GT}$ jouait un rôle actif dans le transport de certains acides aminés. Néanmoins, des malades ne possédant pas de $\gamma$ GT détectable (glutathionurie) ont un transport normal d'acides aminés [27, 28]; il en est de même pour des cellules dont l'activité $\gamma \mathrm{GT}$ a été complètement inhibée par l'acivicine (AT125). La $\gamma \mathrm{GT}$ pourrait donc contribuer à l'entrée de certains acides aminés dans la cellule sans que ce mécanisme soit vital.

La $\gamma$ GT joue un rôle capital dans la détoxification cellulaire par le GSH. Les cellules sont agressées en permanence par des agents toxiques électrophiles résultant principalement de la transformation des xénobiotiques par le cytochrome P450. Le GSH va les neutraliser en les conjuguant par l'intermédiaire de glutathion transférases. Ces conjugués, transformés par une suite de réactions (figure 6) sont éliminés sous forme d'acides mercapturiques [29, 30]. Ces réactions prennent place dans différents tissus - foie, rein, intestin - et peuvent faire intervenir la flore microbienne. La première étape de cette dégradation est assurée par la $\gamma \mathrm{GT}$.

La $\gamma \mathrm{GT}$ participerait également à l'élimination d'ion ammonium.

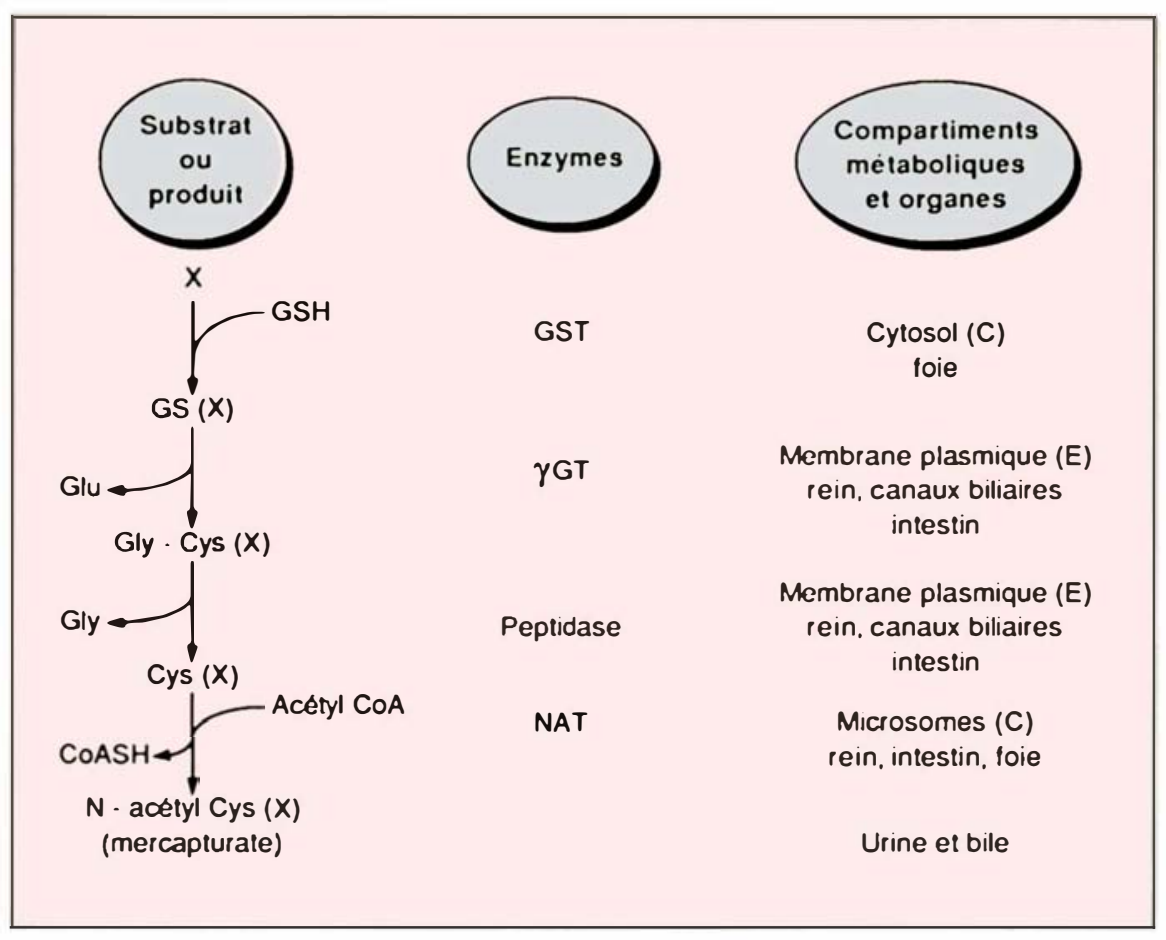

Figure 6. Enzymes impliquées dans la synthèse d'acides mercapturiques. Quatre réactions enzymatiques interviennent dans l'élimination des composés électrophiles toxiques. La première réaction est catalysée par le groupe des glutathion transférases (GST) qui conjugent le GSH aux composés toxiques (X). La deuxième réaction est catalysée par la yGT qui libère l'acide glutamique (Glu) et le dipeptide Cys-Gly conjugué au composé $X[G l y-C y s(X)]$. Ce dernier est hydrolysé par une dipeptidase en Gly et Cys $(X)$. Le produit $C y s(X)$ est acétylé par une acétyltransférase (NAT) en acide mercapturique [ $(\mathrm{N}$-acétyl Cys $(X)]$. Ces réactions peuvent intervenir dans le cytoplasme $(C)$ ou à la surface de la cellule $(E)$. (D'après [19].)

Chez les mammifères, cet ion est éliminé par l'intermédiaire du cycle de l'urée. La perturbation de ce cycle entraîne une augmentation d'ion ammonium qui est transformé en glutamine par la glutamine synthétase en présence de glutamate. Cette glutamine en excès se retrouve dans le fluide cérébrospinal et produit de profondes perturbations neurologiques.

Or, si la glutamine est essentiellement hydrolysée par une glutaminase mitochondriale, elle le serait également par la $\gamma \mathrm{GT}$ rénale, favorisant ainsi une élimination d'ion ammonium dans les urines. Ce point est encore controversé car l'inhibition irréversible et pratiquement complète de la $\gamma \mathrm{GT}$ par l'acivicine ou AT 125 a des effets contradictoires sur la production d'ion ammonium dans les urines. De plus, selon certains auteurs, l'affinité de la $\gamma \mathrm{GT}$ pour la glutamine serait trop faible pour que cette enzyme ait une contribution significative dans l'hydrolyse de la glutamine [31, 32].

La $\gamma \mathrm{GT}$ participe aussi au métabolisme des leucotriènes. Ces composés sont formés à partir de l'acide arachidonique. Trois d'entre eux - le LTC4, le LTD4 et le LTE4 - constituent les slow reacting substances of anaphylaxis (SRSA) et jouent un rôle capital dans la bronchoconstriction. Le LTC4 est formé à partir du LTA4 par conjugaison au GSH. La $\gamma$ GT catalyse l'hydrolyse du LTC4 en LTD4, mais également la transpeptidation inverse. Le LTD4 est transformé en LTE4 par une dipeptidase. La $\gamma \mathrm{GT}$ intervient donc dans le rapport LTC4/LTD4. Il faut signaler que ces différents leucotriènes et le GSH ont une affinité du même ordre de grandeur pour la $\gamma \mathrm{GT}$. Ceci est un argument en faveur du rôle physiologi- 


\section{RÉFÉRENCES}

28. Pellefigue F, Debrohun Butler J, Spielberg SP, et al. Normal aminoacid utptake by cultured human fibroblasts does not require $\gamma$-glutamyl transpeptidase. Biochem Biophys Res Commun 1976 : 73 : 997-1002.

29. Tate S. Enzymes of mercapturic formation. In : Jakoby N, ed. Enzymatic basis of detoxification. New York: Academic Press, 1980 : $95-119$

30. Reed DJ, Meredith MJ. Glutathione conjugation systems and drug disposition. In : Roe DA, Campbell TC, eds. Drugs and Nutrients: The interactive effect. New York, Basel : Dekker M. Inc. 1982; 179-224.

31. Welbourne TC, Dass PD. $\gamma$-glutamyl transpeptidase contribution to renal ammoniagenesis in vivo. Pflug Arch 1988 ; 411 : 573-

32. Curthoys NP. Role of $\boldsymbol{\gamma}$-glutamyl transpeptidase in the renal metabolism of glutathione. Mineral Elect Metab 1983 ; 9 : 236-45.

33. Morris HR, Taylor GW, Jones CM, et al. Slow reacting substance (leukotrienes): enzyme involved in their biosynthesis. Proc Nall Acad Sci USA 1982; 79 : 4838-42.

34. Anderson ME, Allison RD, Meister A. Interconversion of leukotriene catalyzed by purified $\boldsymbol{\gamma}$-glutamyl transpeptidase: concomitant formation of leukotriene D4 and $\boldsymbol{\gamma}$ glutamyl amino acids. Proc Natl Acad Sci USA 1982 ; 79 : 1088-91. que de la $\gamma \mathrm{GT}$ dans cette réaction métabolique. Il semble que le LTD4 soit plus efficace que le LTC4 pour induire des bronchoconstrictions ainsi que des contractions des muscles lisses de l'intestin. Ces deux leucotriènes ont des récepteurs membranaires distincts et il restera à prouver l'importance que peut avoir la modification de la balance LTC4/LTD4 par la $\gamma \mathrm{GT}[33,34]$.

En conclusion, la $\gamma \mathrm{GT}$ est un bon marqueur de certaines maladies hépatiques. Néanmoins, ceux qui s'intéressent à la $\gamma \mathrm{GT}$, tant sur le plan clinique que fondamental, doivent garder à l'esprit que cette protéine peut être une enzyme clé de certaines voies métaboliques comme le métabolisme interorgane du GSH, la détoxification cellulaire, le métabolisme de la glutamine ou celui des leucotriènes. Les modifications d'activité de la $\gamma \mathrm{GT}$, observées au cours de différentes maladies, ne doivent pas uniquement être analysées en terme de marqueur sérique, mais également comme une cause possible de modulation du métabolisme du GSH et de ses dérivés

\section{TIRÉS A PART}

G. Guellaën.

\section{Summary}

Physiological role of $\gamma$-glutamyl transpeptidase

$\gamma$-glutamyl transpeptidase $(\gamma \mathrm{GT})$ is mainly known as a marker of hepatic alterations and its function in the cell metabolism is often underestimated. $\gamma \mathrm{GT}$ is the only enzyme able to hydrolyze the $\boldsymbol{\gamma}$-glutamyl moiety of glutathione and its derivatives. It participates to the glutathione cycle that occurs in the liver or between this tissue and other organs. $\gamma \mathrm{GT}$ catalyses the initial degradation step of glutathione conjugates leading to the formation of mercapturic acids. This enzyme is also able to interconvert leukotrienes $\mathrm{C} 4$ and $\mathrm{D} 4$, thus regulating the equilibrium between both of these compounds. This enzyme might favor aminoacids transport but this reaction is not crucial for the cell. Its participation to glutamine hydrolysis is still controversial. The changes in $\gamma \mathrm{GT}$ activity observed during different pathological situations must not be analyzed only in terms of hepatic alterations, but also as an important modulator of the glutathione metabolism and its derivatives.

\section{ATELIERS DE FORMATION INSERM}

Les ateliers INSERM constituent de la part de I'Institut National de la Santé et de la Recherche Médicale un effort sans précédent de formation des chercheurs français aux techniques de pointe, celles qu'il faut posséder pour figurer honorablement dans la rude émulation internationale dans le domaine des sciences biologiques. S'adressant à des scientifiques dejà confirmés, engagés dans des recherches pouvant bénéficier des avancées méthodologiques qui sont l'objet des ateliers, ces réunions comportent deux parties: une phase I, qui fait le point sur le domaine méthodologique traité, et une phase II, de maîtrise technique, qui permet à un petit groupe de se familiariser avec la réalité des technologies présentées. Ce sont les plus grands spécialistes français et internationaux qui, dans la phase I, présentent des conférences couvrant l'ensemble du champ de l'atelier et, dans la phase II, conduisent les travaux des stagiaires. Les ateliers INSERM 1990 abordent différents domaines des techniques de recombinaison d'ADN, de transfert de gène dans les cellules et les organismes et d'analyse des anomalies génétiques. L'exploration de la transduction des signaux perçus à la membrane cellulaire et des modifications précoces induites est abordée dans deux ateliers consacrés à l'étude des protéines $\mathrm{G}$, des seconds messagers et des potentiels membranaires. Les plus récentes possibilités de l'étude des cellules par cytométrie de flux et par microscopie associée aux marquages immunocytochimiques feront enfin également l'objet de deux ateliers. C'est avec enthousiasme que médecine/sciences participe à la présentation de ces ateliers et continuera de vous informer sur leur organisation et leur déroulement : c'est là une initiative qui procède de la même analyse que celle qui a présidé à la création de notre revue: de la diffusion la plus large possible de l'information de haut niveau et du savoir-faire dépendent les chances que nous avons de l'emporter, c'est-à-dire que de notre communauté scientifique sortent en quantité croissante des résultats faisant avancer les connaissances et les concepts, et par conséquent les possibilités de « mieux faire ^ dans le domaine de la médecine comme dans celui des biotechnologies en général.

Axel Kahn Rédacteur en chef 